\title{
Dissociation between unconscious motor response facilitation and conflict in medial frontal areas
}

\author{
Kevin D'Ostilio ${ }^{1}$ and Gaëtan Garraux ${ }^{1,2}$ \\ ${ }^{1}$ Movere Group, Cyclotron Research Center, University of Liége, Liége, Belgium \\ 2Department of Neurology, University Hospital Center, Sart Tilman B30, 4000 Liège, Belgium
}

Keywords: cingulate, fmri, motor, priming, subliminal, unconscious

\begin{abstract}
Masked prime tasks have shown that sensory information that has not been consciously perceived can nevertheless modulate behavior. The neuronal correlates of behavioral manifestations of visuomotor priming remain debated, particularly with respect to the distribution and direction (i.e. increase or decrease) of activity changes in medial frontal areas. Here, we predicted that these discrepant results could be accounted for by two automatic and unconscious processes embedded in this task: response conflict and facilitation. We used event-related functional magnetic resonance imaging (fMRI), as 24 healthy participants had to respond, as fast as possible, to a target arrow presented immediately after a subliminal masked prime arrow. There were three experimental conditions defined by the prime-target relationship: compatible, incompatible, and neutral. The classical visuomotor priming effect was reproduced, with relatively longer reaction times (RTs) in incompatible trials. Longer RTs in incompatible than in neutral trials were specifically associated with stronger blood oxygen level-dependent (BOLD) activity in a conflict-related network comprising the anterior cingulate cortex and right frontal associative areas. Motor response facilitation as shown by shorter RTs in compatible than in neutral trials was associated with reduced activation in a motor preparation network including the medial and lateral premotor cortices, as a result of the repetition suppression of the fMRI BOLD signal. The present results provide new insights into automatic and unconscious visuomotor priming processes, suggesting an involvement of either a cognitive or motor network, depending on the prime-target relationship.
\end{abstract}

\section{Introduction}

Making a movement in response to stimuli from our environment is often described as being totally conscious and controlled. However, in some situations, movements are not always consciously wished for. There are many motor control disorders in which the patient cannot abstain from making undesirable movements, as if they were automatically activated without the possibility of being stopped. Such an unconscious and automatic activation of the motor response system does not necessarily require stimuli to be consciously perceived. In the 1970s, researchers reported the case of a patient with blindness secondary to primary visual cortex lesion who could continue to differentiate, with a rather good accuracy, two stimuli despite the fact that he claimed not to see them (Weiskrantz et al., 1974). This unexpected phenomenon of 'blindsight' was one of the first pieces of evidence suggesting the existence of a direct perceptuomotor link that allows visual information to directly activate motor responses without conscious processing of the stimulus (Neumann, 1990). This hypothesis was later supported by experiments on healthy subjects that used subliminal priming experiments involving various visual stimuli, ranging from geometric shapes (Neumann \& Klotz,

Correspondence: Dr Gaëtan Garraux, ${ }^{2}$ Department of Neurology, as above.

E-mail: ggarraux@ulg.ac.be

Received 22 August 2011, revised 5 October 2011, accepted 18 October 2011
1994) or words (Dehaene et al., 1998) to more simple stimuli such as arrows (Eimer \& Schlaghecken, 1998). In these visuomotor tasks, participants are typically asked to make a rapid button press in response to the display of a target stimulus to which it has been previously assigned. Unbeknown to the subjects, each target stimulus is immediately preceded by a brief masked prime stimulus presented below the threshold of awareness. Behaviorally, the effect is similar to what is typically observed in classic conflict tasks such as the Eriksen flanker task (EFT) (Eriksen \& Eriksen, 1974), subjects responding faster and/or making fewer errors when the primeinduced and target-induced responses are congruent than when they differ.

In functional magnetic resonance imaging (fMRI) or electrophysiological experiments, this difference in reaction time (RT) has been related to activity changes in the motor cortices (Dehaene et al., 1998; Eimer \& Schlaghecken, 1998; Praamstra \& Seiss, 2005), suggesting that the activation induced by a masked prime spreads to the regions involved in motor response execution. However, the involvement of other cortical brain regions in subliminal motor priming has not yet been clearly investigated. Unlike previous work, the present study was designed to separate two distinct processes at both behavioral and neuronal levels: response facilitation and conflict. We reasoned that the RT difference between compatible and incompatible trials resulted from both facilitation (i.e. in compatible trials) and conflict (i.e. in 
incompatible trials) effects. Here, these effects were dissociated by incorporating neutral trials in the experimental paradigm, that is, using masked prime stimuli that have not been associated with any motor response. After assessing the main difference between compatible and incompatible conditions at both the behavioral and fMRI levels, we estimated the facilitation and conflict effects by comparing compatible and incompatible conditions with the neutral condition, respectively. On the basis of the results of a recent fMRI experiment from our group (D'Ostilio \& Garraux, 2011), we predicted that the facilitation effect would be mediated, at least in part, in the medial premotor cortex (MPMC). Because, in compatible trials, the masked prime and target stimuli are identical, we hypothesized that activity in this region would decrease as a result of repetition suppression (RS) of the fMRI blood oxygen level-dependent (BOLD) signal. This effect has been previously described in motor processing (Grafton \& Hamilton, 2007), word reading (Dehaene et al., 2001), mathematical cognition (Salimpoor et al., 2010), and even speech perception (Kouider et al., 2010). In the conflict effect, we predicted that unconscious priming could interact with response selection and influence high-level frontal areas. Therefore, we expected greater activity in incompatible trials in the anterior cingulate cortex (ACC), a key region of conflict monitoring.

\section{Material and methods}

\section{Subjects}

Twenty-four healthy right-handed volunteers (six men), with no diagnosed psychological or neurological disorders, were recruited from the university community. Their ages ranged from 18 to 27 years, with a mean of $21 \pm 2$ years. The study was approved by the Ethics Committee of the Faculty of Medicine, University of Liège, Belgium. All volunteers gave their written informed consent prior to their inclusion in the study, and were paid for their participation.

\section{Task procedure}

The task paradigm was adapted from that reported in Eimer \& Schlaghecken (1998). In this visuomotor task, subjects are asked to press a response button as accurately and as quickly as possible with their left or right hand in response to the presentation of a left-pointing or right-pointing arrow. In each trial, this target stimulus is briefly preceded by a prime stimulus (Fig. 1). Whereas the target stimulus is displayed long enough to be consciously perceived, the prime stimulus is not, because its display is short (i.e. $17 \mathrm{~ms}$ ) and is immediately followed by a backward mask. A high response conflict between left and right manual responses is expected when the prime and target arrows point in opposite directions (incompatible trials) (Aron et al., 2003).

In each trial, the following stimuli were sequentially displayed on a screen: fixation point, prime, mask, and target stimulus. Each trial started with a central fixation point. Its display was pseudo-randomly jittered between 1500 and $3000 \mathrm{~ms}$. The fixation point display was immediately followed by a blank screen and prime-arrow stimulus, sequentially presented for 300 and $17 \mathrm{~ms}$, respectively. Then, a backward mask and two double target arrows appeared for $100 \mathrm{~ms}$. The mask consisted of 30 randomly oriented lines covering a rectangular area centered on the prime display area on the center of the screen. A new mask was constructed on each trial. The two double arrows, which appeared on both sides of the mask, were imperative stimuli prompting participants to make a rapid button press with their left or right index finger according to the direction of these target arrows.

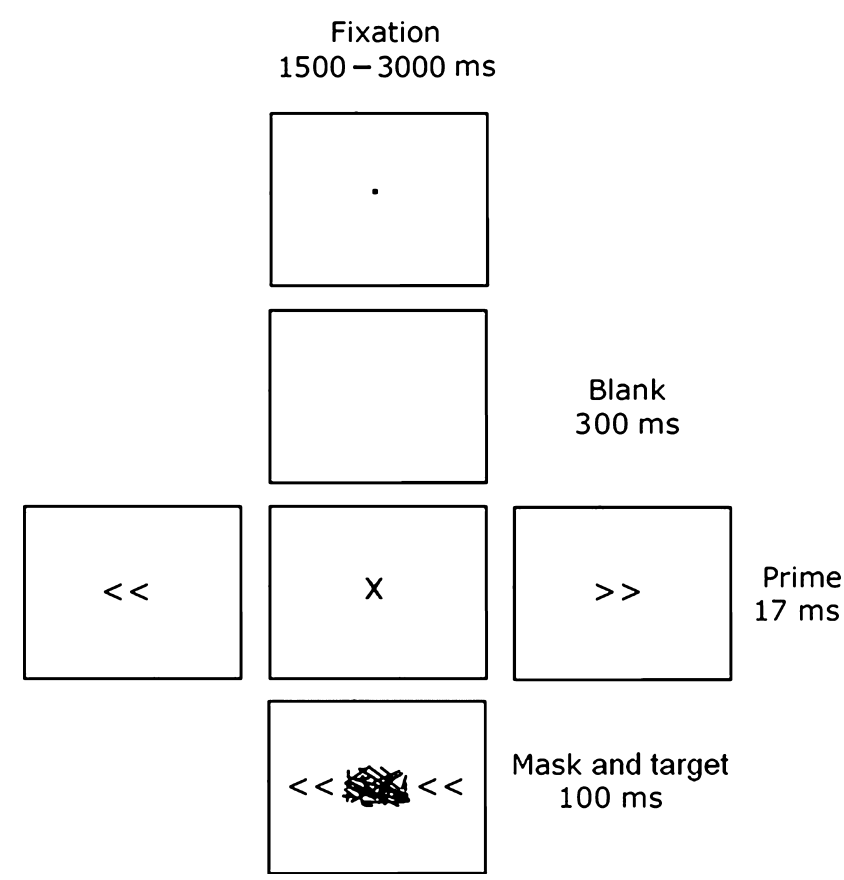

FIG. 1. Behavioral paradigm. Example of compatible (left panel), neutral (middle panel) and incompatible (right panel) response trials. The targets appeared together with the mask, directly after the prime presentation. The only difference in the three conditions was the physical properties of the 17-ms unperceived prime.

Each subject was trained on a practice block of 30 trials outside the magnetic resonance imaging (MRI) scanner. The experiment included 40 compatible trials, 40 incompatible trials, 40 neutral trials (the prime stimulus was an ' $\mathrm{X}$ ' sign), 40 null events (fixation point instead of a trial), and 80 no-response trials as part of another study (D'Ostilio \& Garraux, 2011). The outcome measures were the RT and accuracy in response to the target stimuli. Stimuli were grouped in blocks of 36 trials presented in random order. After the completion of each block, subjects were provided with a 20 -s rest period, during which the mean global RT during the last performed block was displayed.

The level of prime perception during the subliminal conflict task was assessed by three independent measures. All participants were first interviewed at the end of the main fMRI study. Second, participants performed a prime identification task in the MRI scanner for assessment of the level of prime perception as a function of the prime display duration. In each trial, the stimuli (left-pointing or rightpointing arrow) and their temporal sequence of presentation were the same as in the main experiment, with the exception that the target stimulus was a question mark displayed from 1 to $1.7 \mathrm{~s}$ after the mask. This question mark prompted the participants to make a response with the left or right hand within $3 \mathrm{~s}$, according to the direction of the arrow stimulus. No emphasis was placed on RT. Prime display duration varied according to a one-down/two-up staircase procedure (Schlaghecken \& Eimer, 2002; Schlaghecken \& Sisman, 2006). The task always started with a $167-\mathrm{ms}$ display trial. In the following trials, prime display was shortened by steps of $17 \mathrm{~ms}$ whenever participants gave a correct response, and prolonged by $34 \mathrm{~ms}$ after an incorrect response. In trials where the prime did not reach the level of awareness, participants were invited to guess the response that they felt to be the most accurate. We calculated the overall mean prime duration and percentage of correct responses at $17 \mathrm{~ms}$ (Schlaghecken \& Sisman, 2006). Third, a second identification task was administered 
in the MRI scanner to 10 young healthy subjects (four men, aged $27 \pm 3$ years) who did not participate in the fMRI experiments. Here, the task display was exactly the same as in the main experiment (i.e. with target arrows), but participants were asked to guess which prime stimulus was presented before the mask (left-pointing arrow, rightpointing arrow, or neutral stimulus). They performed 120 trials (40 for each stimulus type). We calculated the percentage of correct responses in each of the three conditions.

Visual stimuli were generated and subject responses recorded with a personal computer using COGENT 2000 cognitive interface software (Wellcome Department of Imaging Neuroscience, London, UK) implemented in MATLAB 6.1 (Mathworks, Sherborn, MA, USA).

\section{Imaging data acquisition}

BOLD fMRI data were acquired on a 3-T scanner (Siemens, Allegra, Erlangen, Germany) with a T2*-sensitive gradient echoplanar imaging (EPI) sequence (repetition time, $1170 \mathrm{~ms}$; echo time, $30 \mathrm{~ms}$, flip angle, $90^{\circ}$; matrix size, $64 \times 64 \times 20$; voxel size, $3.4 \times 3.4 \times 5 \mathrm{~mm}^{3}$ ). Twenty 5 -mm-thick slices were acquired, covering nearly the whole brain. For each session, the first eight volumes, acquired before stimulus presentation, were discarded to allow for $\mathrm{T} 1$ saturation effects. Head movement was minimized by restraining the subject's head with a vacuum cushion. Stimuli were displayed on a screen positioned at the rear of the scanner, which the subject could comfortably see through a mirror mounted on the standard head coil. High-resolution structural images were obtained with a T1-weighted 3D MDEFT sequence (repetition time, $7.92 \mathrm{~ms}$; echo time, $2.4 \mathrm{~ms}$; flip angle, $15^{\circ}$; matrix size, $224 \times 256 \times 176$; voxel size, $1 \times 1 \times 1 \mathrm{~mm}^{3}$ ).

\section{Behavioral data analysis}

We computed the mean individual RT and accuracy in all conditions. Trials with inaccurate response or RT $>1 \mathrm{~s}$ were excluded from the RT and fMRI data analysis. As in previous studies (Schlaghecken \& Maylor, 2005; Seiss \& Praamstra, 2006), we estimated the compatibility effect by comparing the group mean RT between compatible and incompatible trials. Comparisons with neutral trials were then calculated in order to isolate the conflict effect (incompatible - neutral) and the facilitation effect (compatible - neutral). Statistical significance was assessed with repeated-measures ANOVA on mean RT with the condition as an intrasubject factor (Statistica 8; StatSoft, France).

In the identification tasks, $t$-tests were used to compare the mean accuracy with chance performance $(50 \%$ in the first identification task and $33 \%$ in the second one). We also calculated the mean prime duration, by averaging all prime durations for each subject after having discarded the first 10 trials, as this is the minimum number of steps needed to reach the 17-ms performance limit (e.g. Schlaghecken \& Sisman, 2006). This analysis allowed us to obtain a converging value of the display duration at which participants consciously perceive the prime on average.

\section{Imaging data processing}

Data were preprocessed and analyzed with SPM8 (Wellcome Department of Imaging Neuroscience; http://www.fil.ion.ucl.ac.uk/spm) implemented in MATLAB 7.4.0 (Mathworks). For each participant, we first applied a slice-timing correction to compensate for the staggered order of slices acquired by EPI. The BOLD time series was then spatially realigned by the use of iterative rigid body transformations, which minimize the residual sum of squares between the first and subsequent images. Imaging data from one participant were excluded from data analysis because of significant head movement artefacts. The mean EPI image was spatially coregistered to the anatomical MRI image, and coregistration parameters were applied to the realigned BOLD time series. Individual anatomical magnetic resonance images were spatially normalized into Montreal Neurological Institute (MNI) space (http://www.bic.mni.mcgill.ca) with the unified segmentation approach, and the normalization parameters were subsequently applied to the individually coregistered BOLD time series, which was then resliced to a voxel size of $2 \times 2 \times 2 \mathrm{~mm}$, and finally smoothed with a $6-\mathrm{mm}$ full width at half-maximum Gaussian kernel.

\section{fMRI data analyses}

All main experimental trials were modeled as single events timelocked on the target stimulus display onset. Each event was convolved with a canonical hemodynamic response function and its time and dispersion derivatives. In addition, the statistical model included six additional regressors representing the realignment parameters from the rigid body transformation step. A high-pass filter with a cut-off period of $128 \mathrm{~s}$ was applied in order to remove the low-frequency drifts from the time series. Serial autocorrelations were estimated with a restricted maximum likelihood algorithm with an autoregressive model of order 1 (+ white noise). Parameter estimates and variance were derived voxel-by-voxel, and the main effect of each condition was assessed with $t$-contrasts.

We performed three-first-level analyses modeling relative activity increases during compatible, incompatible or neutral trials as compared with null events. We covaried for RT in order to ensure that activity differences between experimental conditions were only attributable to the effect of interest. In the context of a random-effect model, in which a single measurement is obtained from each subject (Penny et al., 2003), these individual contrast images from level one analyses were entered in a second-level analysis. As in the behavioral data analysis, the comparison between compatible, incompatible and neutral trials was performed with an ANOVA. This analysis appropriately accounts for intersubject variability, and tests whether or not the population from which our set of subjects is drawn possesses the hypothesized effects. Group results were characterized in terms of the probability that the difference in magnitude value in a given voxel could occur by chance under the null hypothesis.

In the ANOVA, we examined the effect of each condition (as compared with baseline) at a peak threshold of $P<0.001$, uncorrected. Following our main hypothesis (see Introduction), we next dissociated the conflict effect from the facilitation effect by using the contrasts 'incompatible minus neutral' and 'neutral minus compatible', respectively. The results of these two analyses were masked inclusively by the statistical parametric maps $(P<0.001$ uncorrected $)$ modeling the effect of incompatible and neutral conditions, respectively (see above). Subliminal priming effects are very small, and corresponding brain activations are usually hard to detect. For that reason, any results surviving a peak threshold of $P<0.005$ uncorrected, with an extent threshold of 10 voxels in these areas, were considered to be significant (Kouider et al., 2010). Statistical significance was also restricted by small volume corrections (SVCs) in our $a$ priori regions of interest (i.e. the ACC for the conflict effect and the MPMC for the facilitation effect). The SVC was computed by using a familywise error correction for multiple comparisons in a sphere of diameter $10 \mathrm{~mm}$ centered on published coordinates of the ACC (MNI: 10, 24, 34) (Lau et al., 2006) and the peak voxel given by our 
previous experiment examining the neural correlates of automatic motor activation with no-response conditions performed on the same sample of subjects (MNI: 8, -2, 62) (D'Ostilio \& Garraux, 2011). We also tentatively reported activations in other brain regions, but those results will not be extensively discussed.

\section{Results}

\section{Behavioral data}

\section{Identification tasks}

We were confident that the primes were not consciously perceived. At interview, after fMRI, participants all denied having perceived any stimulus immediately preceding the mask stimulus during the subliminal priming task. This impression was confirmed by the results of identification task analyses. For the participants of this experiment, the mean prime duration was $35.62 \mathrm{~ms}$. They responded, on average, to 36 stimuli displayed at $17 \mathrm{~ms}$, as opposed to 44 for the other prime durations. In a staircase task, if they had really seen the prime, they should have more responses to the 17 -ms prime stimulus than to other prime duration stimuli. In order to enhance the power of the statistical analysis, we added the results of 23 supplementary subjects who performed the task in the same conditions. When the prime arrow was presented for $17 \mathrm{~ms}$, response accuracy did not differ significantly from chance level (mean percentage correct responses at $\left.17 \mathrm{~ms}=54.7 \%, t_{46}=1.46, P>0.1\right)$ (Fig. 2A). A second identification task, with the same timing and stimuli as the main task, administered in the MRI scanner to 10 young healthy subjects who did not participate in the fMRI experiment, also demonstrated that the masked prime displayed at $17 \mathrm{~ms}$ was subliminal (mean percentage correct responses at $17 \mathrm{~ms}=31.7 \%, t_{9}=0.79, P>0.4$ ) (Fig. 2b). Altogether, these results support the view that prime stimuli were unlikely to be consciously perceived by the participants during the main task.

\section{Main task}

The main effect of condition was statistically significant $\left(F_{2,46}=7.72\right.$, $P=0.001)$ in such a way that, relative to neutral trials (mean RT $=$ $375 \pm 38 \mathrm{~ms}$ ), RT was shorter in compatible trials (mean RT $=369 \pm$ $38 \mathrm{~ms}$ ) and longer in incompatible trials (mean RT $=383 \pm 30 \mathrm{~ms}$ ) (unilateral tests: compatibility effect $=14$; incompatible $>$ compatible, $t_{23}=3.42, P=0.001$; incompatible $>$ neutral, $t_{23}=2.38, P=$ 0.013 ; neutral $>$ compatible, $\left.t_{23}=1.90, P=0.035\right)$. The results are illustrated in Fig. 3. Accuracy rate showed a similar tendency, but no statistical inference was made, given the small number of errors.

\section{Imaging data}

Figure 4 shows significant differences in BOLD signal for the compatible, neutral and incompatible conditions in comparison with the baseline condition (null events). The pattern of activation revealed an involvement of cognitive control regions, notably the ACC, during incompatible trials, but reduced activity in the MPMC during compatible trials. The main effect of the ANOVA is reported in Table S1. To examine the compatibility effect-related activity, we contrasted the BOLD response between incompatible and compatible trials. We found stronger activity in our a priori defined region, the ACC ( $x=10, y=26, z=28, T=4.89, P=0.000003)$, and also in two related high-level cognitive areas, the right dorsolateral prefrontal cortex (DLPFC) $(x=34, y=48, z=38, T=3.30, P=0.0007)$ and inferior parietal cortex $(x=66, \quad y=-30, \quad z=38, \quad T=3.69$,
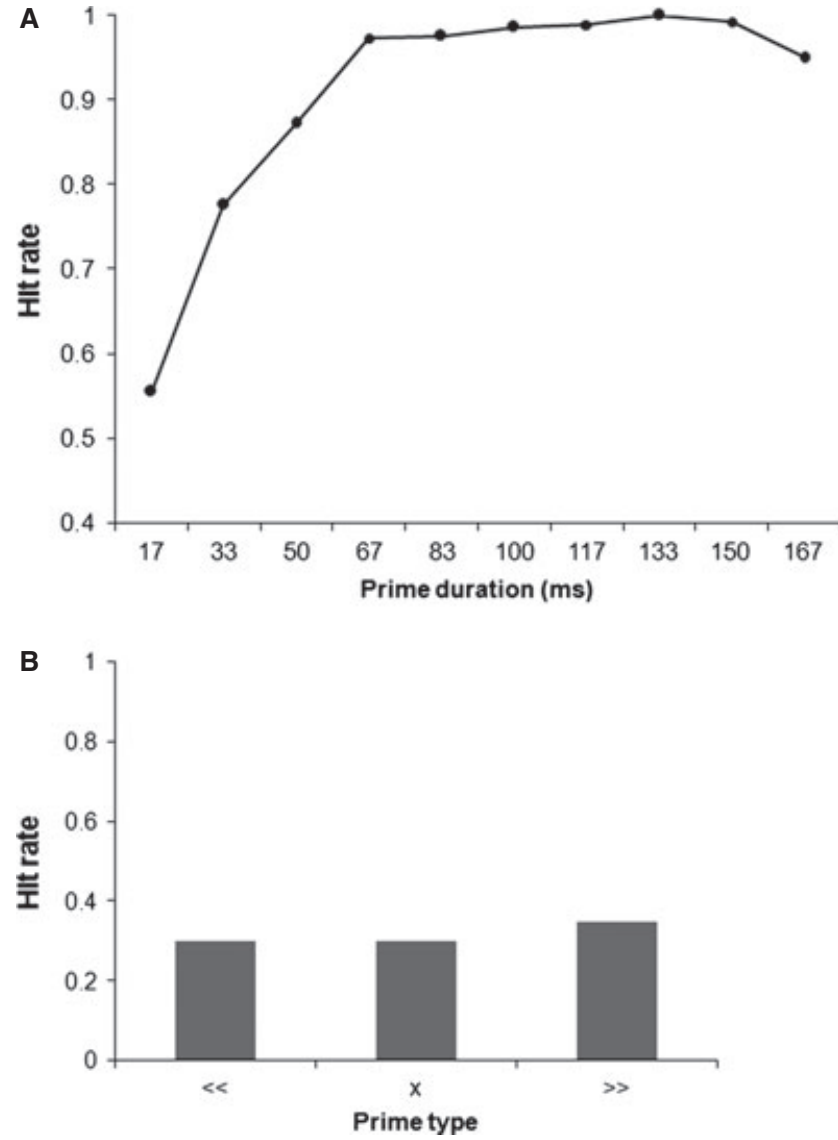

FIG. 2. (A) Accuracy rate in the first prime identification task, plotted separately for each prime duration. Participants were only unable to discriminate the arrow direction for the $17-\mathrm{ms}$ prime (chance-level performance). (B) Accuracy rate in the second prime identification task (10 participants) for each stimulus type (left arrow, 30.2\%; neutral stimulus, 30\%; right arrow, 34.7\%).

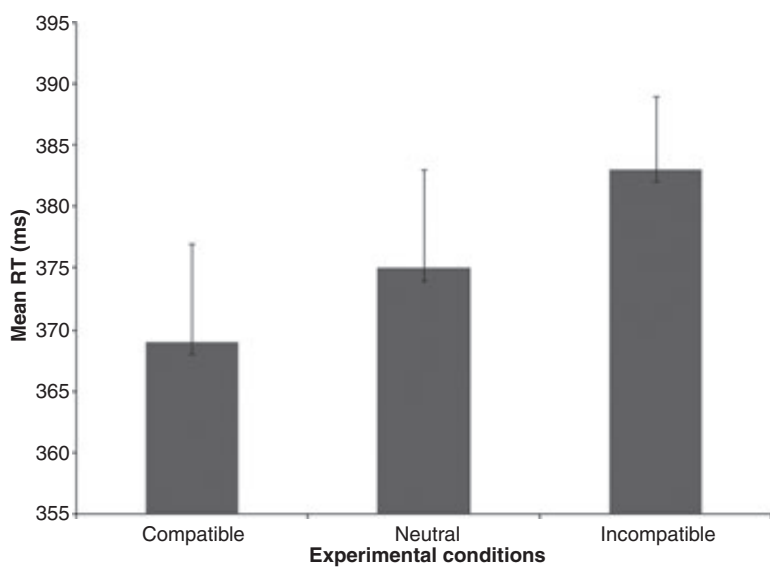

FIG. 3. Behavioral results. Mean RTs in the three experimental conditions. The RT in incompatible trials was significantly longer than that in the other two conditions. Vertical bars represent standard errors.

$P=0.0002)$. The complete results for this contrast are reported in Table S2. We isolated the conflict effect by comparing incompatible trials with neutral trials (Table 1; Fig. 5). The facilitation effect was assessed by contrasting neutral trials with compatible trials. We found smaller activation in compatible trials in the medial and dorsal 


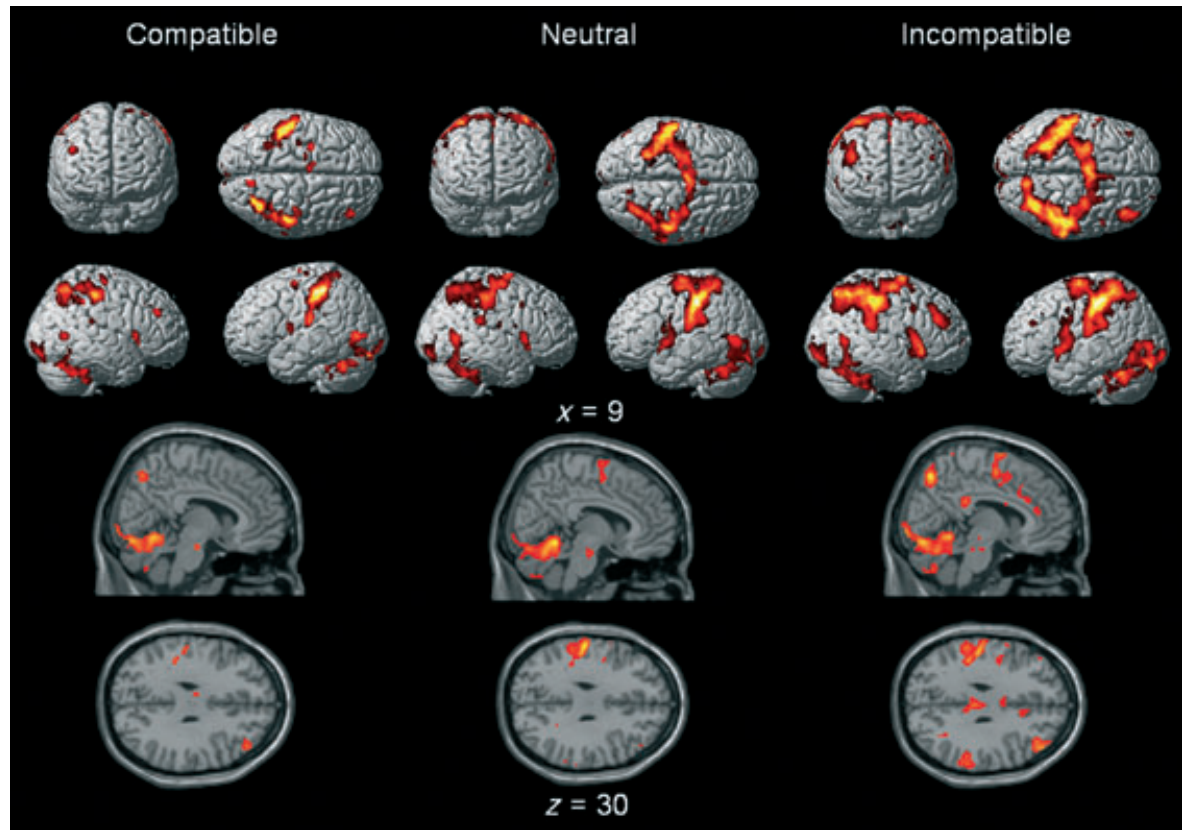

FIG. 4. BOLD activations in the three experimental conditions (compatible, neutral, and incompatible) minus baseline, displayed at a statistical threshold of $P<0.001$ uncorrected. In incompatible trials, the intensity and extent of the activation were higher in the ACC and the DLPFC. In compatible trials, activity in the premotor system was reduced.

TABLE 1. Maxima within all brain regions showing BOLD signal increases in the conflict effect

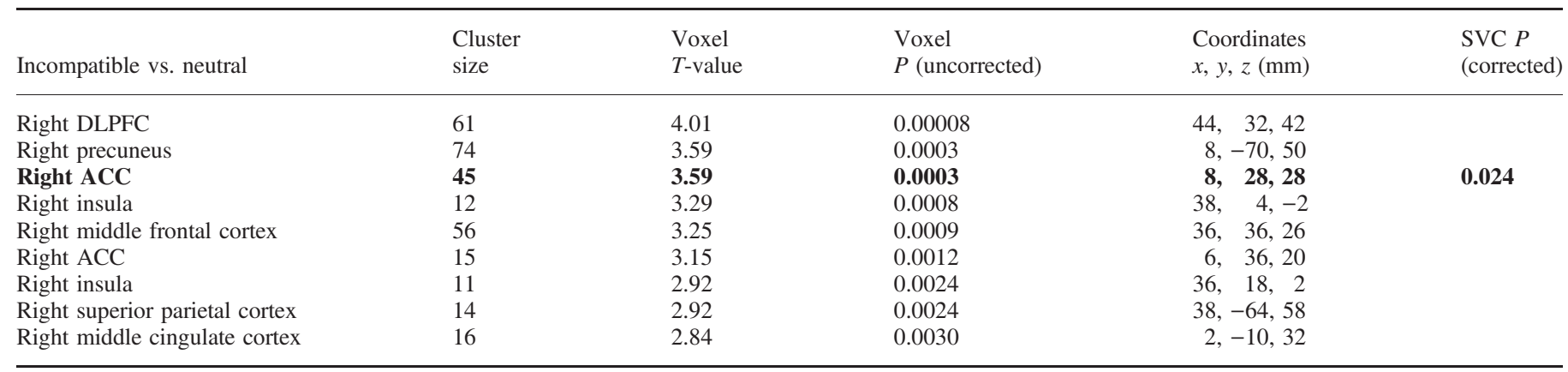

premotor cortex as a result of RS of the BOLD signal. The results are reported in Table 2 and Fig. 5.

\section{Discussion}

In this event-related fMRI experiment, the BOLD signal was measured in healthy individuals under three main experimental conditions that differed only by the physical properties of a stimulus presented below the threshold of awareness (Fig. 1). The effect of these stimuli was nevertheless strong enough to induce differences in RT (Fig. 3) and BOLD signal (Fig. 4) between conditions. As in previous studies (Botvinick et al., 1999; Seiss \& Praamstra, 2004; Lau et al., 2006), the compatibility effect was measured by comparing RT differences between compatible and incompatible conditions. In agreement with findings in conflict tasks elicited by supraliminal stimuli such as in the EFT, we observed a longer RT and stronger ACC activation in incompatible trials. In addition, other high-level brain areas, such as the right DLPFC and right parietal cortex, were also more strongly activated in incompatible trials. The main prediction of the present study was that the difference between incompatible and compatible trials might be subserved by the combination of several processes, namely the automatic activation of competing motor plans, response conflict (i.e. in incompatible trials), and response facilitation (i.e. in compatible trials).

We have evidence suggesting that unconscious conflict processing was indeed an important component embedded in the task. This was supported by the results of the comparison with neutral trials defined by the presence of a prime stimulus that has not been previously associated with any specific motor response. The RT was longer in incompatible than in neutral trials (Fig. 3). This RT difference was associated with stronger activations in the ACC and DLPFC in incompatible than in neutral trials, suggesting that these regions were specifically involved in unconscious and automatic conflict processing (Fig. 5). As conflict processing is one of the most important functions subserved by the frontal lobes, our result may have important implications for the role of the frontal lobes in unconscious cognitive control in general. 


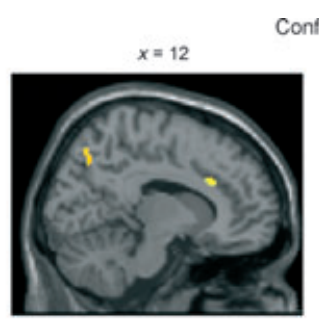

$x=38$

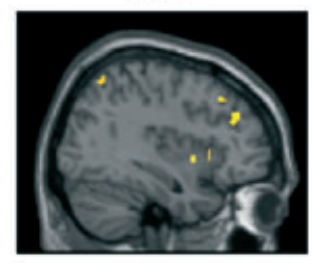

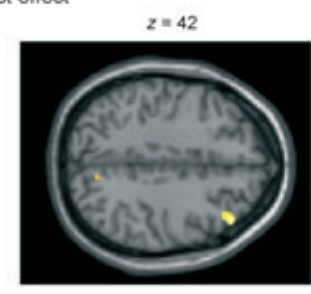

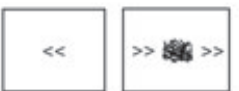

$-$
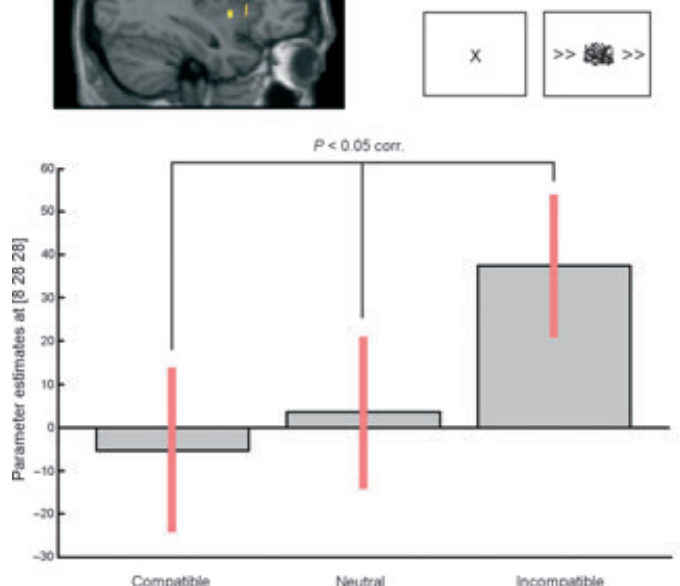

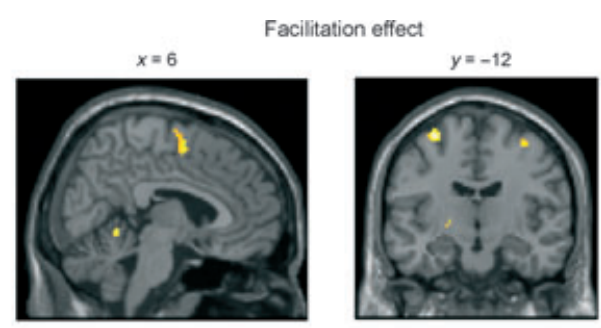

$z=64$
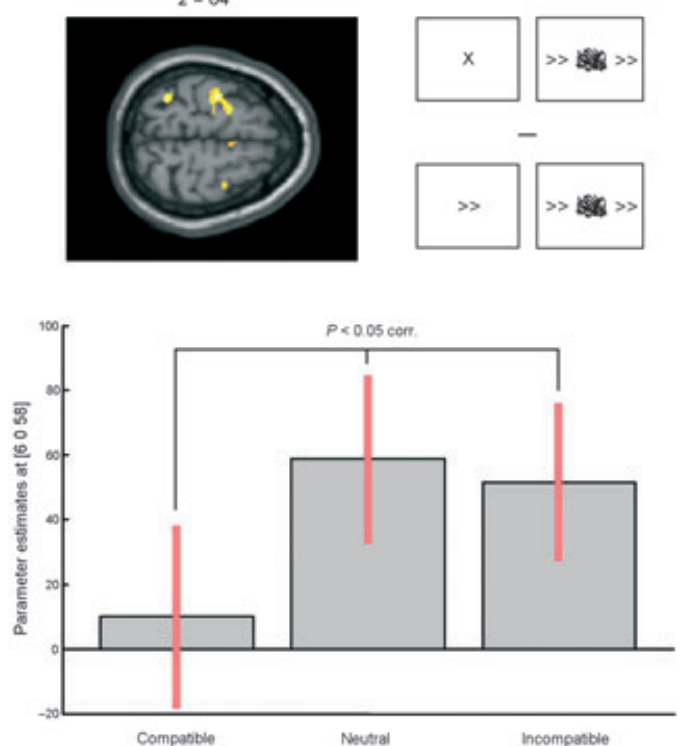

FIG. 5. Comparison of BOLD signal changes during incompatible trials vs. neutral trials (left panel) and neutral trials vs. compatible trials (right panel). The conflict effect was related to high-level activation of brain areas, notably the ACC (left plot) and the DLPFC, whereas the facilitation effect was subserved by reduced activation in a motor preparation network including the MPMC (right plot). Results are displayed at a peak threshold of $P<0.005$ uncorrected, an extent threshold of $P<0.005$ uncorrected, and an extent threshold of 10 continuous voxels.

TABLE 2. Maxima within all brain regions showing BOLD signal decreases in the facilitation effect

\begin{tabular}{|c|c|c|c|c|c|}
\hline Neutral vs. compatible & $\begin{array}{l}\text { Cluster } \\
\text { size }\end{array}$ & $\begin{array}{l}\text { Voxel } \\
T \text {-value }\end{array}$ & $\begin{array}{l}\text { Voxel } P \\
\text { (uncorrected) }\end{array}$ & $\begin{array}{l}\text { Coordinates } \\
x, y, z(\mathrm{~mm})\end{array}$ & $\begin{array}{l}\text { SVC } P \\
\text { (corrected) }\end{array}$ \\
\hline Left postcentral gyrus/primary motor cortex & 141 & 4.22 & 0.00004 & $-60,-20,32$ & \\
\hline Left premotor cortex & 179 & 4.00 & 0.00008 & $-20,-4,62$ & \\
\hline Right cerebellum & 26 & 3.96 & 0.00009 & $8,-52,-8$ & \\
\hline Left inferior parietal cortex & 82 & 3.84 & 0.0001 & $-42,-26,44$ & \\
\hline Left superior parietal cortex & 45 & 3.81 & 0.0002 & $-30,-50,62$ & \\
\hline Right premotor cortex & 39 & 3.37 & 0.0006 & $36,-10,60$ & \\
\hline MPMC (right supplementary motor area) & 69 & 3.25 & 0.0009 & $6, \quad 0,58$ & 0.014 \\
\hline MPMC (left supplementary motor area) & 34 & 3.19 & 0.0011 & $-6,-2,52$ & \\
\hline Right supramarginal gyrus & 16 & 3.16 & 0.0012 & $62,-28,34$ & \\
\hline Left thalamus & 10 & 3.04 & 0.0017 & $-20,-14,2$ & \\
\hline
\end{tabular}

The present results help to resolve discrepancies between previous functional imaging studies examining the involvement of the ACC in response conflict when this conflict is not consciously perceived. In agreement with the theory that the prefrontal cortex (Norman \& Shallice, 1986) and even the ACC (Alexander et al., 2007) belong to a supervisory system that is able to guide only conscious controlled actions, Dehaene et al. (2003) did not find stronger ACC BOLD activity in the incompatible condition than in the compatible condition in a subliminal masked prime task. However, this negative result has been questioned, because it cannot be interpreted as a proof of the null hypothesis, and because of the robust ACC activation not only in incompatible but also, unexpectedly, in compatible trials as compared with baseline (Ursu et al., 2008). In an event-related potential study,
Praamstra \& Seiss (2005) identified a candidate N2 component, a negative wave that may originate in the ACC (Klopp et al., 1996; Van Veen \& Carter, 2002; Gajewski et al., 2008), in the conflict condition, but this was interpreted as a response artefact. In our opinion, none of those studies provide enough evidence to reject a role of the ACC and other frontal lobe regions in unconscious conflict. Conversely, the opposite prediction has received indirect support from two other studies. Hughes et al. (2009) observed that the N2 component was modulated as a function of the stimulus compatibility. In that study, one arrow direction was associated with a no-go response, making difficult the interpretation of results as being attributable to a conflict effect or primed inhibition processes. In an fMRI study, Ursu et al. (2008) used a serial response task with an implicit probabilistic 
learning rule, and found that the ACC was more activated in trials that violated the sequence than in trials where stimuli followed the sequence. However, the differential activation in those studies cannot be solely interpreted as a result of an unconscious conflict effect, as several other processes, including motor learning and response to novelty, were likely to be embedded in that task. Unlike these studies, the present study design allowed separation of the conflict effect from other processes embedded in the task.

The processing of unconscious conflict is poorly understood. Some authors argue for reciprocal inhibition processes involving only the motor system (Praamstra \& Seiss, 2005), whereas others suggest involvement of the frontal cognitive system, through attentional neuromodulation (e.g. Sohrabi \& West, 2009). In line with the latter theory, we showed a pattern of activation similar to what happens in traditional interference task such as the Stroop task, the EFT, or the Simon task (Wager et al., 2005; Nee et al., 2007), suggesting that unconscious information can influence the cognitive control system. According to Coulthard et al. (2008a), the right parietal cortex might interact with prefrontal regions to influence response choice when conflicting responses are in competition. The prefrontal cortex is considered to be the highest cortical area, because of its substantial involvement in executive functioning (Collette et al., 2006). Although the activities of these two regions seem to be closely related, they probably have distinct roles. According to current models, the ACC has, rather, an evaluative function (MacDonald et al., 2000) that enables the lateral prefrontal cortex to implement control trough inhibitory mechanisms (Hazeltine et al., 2000; Nee et al., 2007). Taken together, these results, including ours, do not support the prediction that the ACC and other frontal lobe regions are exclusively involved in conscious cognitive control. Our finding therefore suggests that these regions implement processes that finally serve to reduce competition between different response alternatives, whatever the level of perception of stimuli.

Additionally, our results extend those from previous imaging studies showing that certain executive functions, such as stop signal inhibition or performance monitoring, could be unconsciously modulated by frontal brain regions involved in conscious cognitive control frontal processes (Lau \& Passingham, 2007; Van Gaal et al., 2008,2010; Pavone et al., 2009). Van Gaal et al. (2008) demonstrated that a masked no-go stimulus could elicit a frontal inhibitory eventrelated potential that correlated with the slowed responses to these stimuli. More recently, the same authors implemented this paradigm in an fMRI study, whose results corroborated the involvement of frontal inhibitory processes in unconscious cognitive control, especially the inferior frontal cortex and the presupplementary motor area. In another study, Pavone et al. (2009) showed that an unconscious error could produce an even-related negativity potential, which is considered to be be a neural correlate of error processing (Gehring et al., 1993) that is probably generated by the ACC (Taylor et al., 2007).

A role of frontal regions in unconscious conflict is also supported by lesion studies in neurological populations. Patients with damage to the frontal lobes may show delayed responses in incompatible trials on the EFT (Coulthard et al., 2008a). In addition to impaired controlled cognitive processes, these patients also display deficits in unconscious and automatic inhibition mechanisms (Sumner et al., 2007; Coulthard et al., 2008b). This is also in line with behavioral studies performed in another population with frontal lobe dysfunction, Parkinson disease patients, who showed enhanced susceptibility to both conscious (Praamstra et al., 1999; Wylie et al., 2005) and unconscious (Seiss \& Praamstra, 2004, 2006) triggered interference. This impairment might result from the ACC hypoactivation, which is broadly connected to the basal ganglia (Playford et al., 1992).
Apart from the conflict effect, we also isolated the facilitation effect by comparing RT and BOLD signal changes between compatible and neutral trials. The RT was shorter in compatible than in neutral trials (Fig. 3). We found that the medial and lateral premotor cortices were less activated in compatible trials (Fig. 5). This can be explained by an RS phenomenon characterized by a reduction in activity for repeated as compared with new stimuli (Henson \& Rugg, 2003; Horner \& Henson, 2008). Recently, Hamilton \& Grafton (2009) demonstrated that suppression of the BOLD signal could occur across the motor network, especially in the premotor system. Additionally, RS was described in some subliminal priming experiments on word reading with reduced activity in word-processing areas (Dehaene et al., 2001; Kouider et al., 2007). Here, we showed that RS can also occur at a motor level, even when there is no awareness of stimulus repetition. Reduced activation of the thalamus was also found in the compatible condition. This region belongs to cortico-basal ganglia loops involved in motor control, in the same manner as the MPMC (Aron et al., 2009). This finding extends the results of our previous study concerning the neural correlates of automatic motor activation, an adaptive process facilitating efficient interaction with environmental stimuli (D'Ostilio \& Garraux, 2011) that occurred in the MPMC. One of the roles of the MPMC is to contribute to the automatic activation of environmental stimuli strongly associated with a preponderant response, in order to facilitate the corresponding action if the movement must be quickly executed. The present results clearly demonstrate that MPMC activity is modulated by action priming.

In conclusion, these findings provide new insights into unconscious priming processing. The difference in RT between compatible and incompatible trials is explained, respectively, by facilitation and conflict effects mediated by different brain regions. We showed that the facilitation effect was associated with decreased activity in several brain areas, notably the MPMC. This finding is in line with our previous results on automatic motor activation. We also demonstrated that the dorsal ACC and other frontal brain areas were automatically and mechanically activated by any conflict situation, when the conflict was not consciously elicited. This calls into question the classical theories that consider consciousness and cognitive control to be intimately related, as well as the exclusive involvement of the frontal cortex in conscious processing of information. To our knowledge, this is the first time that a clear dissociation between a motor and a cognitive network has been revealed with subliminal masked priming. However, it has been suggested that participants may be aware of different levels of subjective conflict in some priming tasks, in such a way that, even if they did not perceive the prime, they could detect a difference in the perception of task difficulty (Wenke et al., 2010). Therefore, we cannot exclude the possibility that related brain activity reached the threshold of awareness, even if it is driven by subliminal visual stimuli.

\section{Supporting Information}

Additional supporting information can be found in the online version of this article:

Table S1. Maxima within regions showing BOLD signal changes for the main effect of condition.

Table S2. Maxima within regions showing BOLD signal changes for the compatibility effect.

Please note: As a service to our authors and readers, this journal provides supporting information supplied by the authors. Such materials are peer-reviewed and may be re-organized for online delivery, but are not copy-edited or typeset by Wiley-Blackwell. Technical support issues arising from supporting information (other than missing files) should be addressed to the authors. 


\section{Acknowledgements}

K. D'Ostilio and G. Garraux are Doctoral Fellow and Senior Research Associate at the Fonds National de la Recherche Scientifique de Belgique (FRS-FNRS), respectively. This work was supported by FRS-FNRS (Grant 1.5.047.06)

\section{Abbreviations}

ACC, anterior cingulate cortex; BOLD, blood oxygen level-dependent; DLPFC, dorsolateral prefrontal cortex; EFT, Eriksen flanker task; EPI, echoplanar imaging; fMRI, functional magnetic resonance imaging; MNI, Montreal Neurological Institute; MPMC, medial premotor cortex; MRI, magnetic resonance imaging; RS, repetition suppression; RT, reaction time; SVC, small volume correction.

\section{References}

Alexander, M.P., Stuss, D.T., Picton, T., Shallice, T. \& Gillingham, S. (2007) Regional frontal injuries cause distinct impairments in cognitive control. Neurology, 68, 1515-1523.

Aron, A.R., Schlaghecken, F., Fletcher, P., Bullmore, E., Eimer, M., Barker, R., Sahakian, B. \& Robbins, T. (2003) Inhibition of subliminally primed responses is mediated by the caudate and thalamus: evidence from fMRI and Huntington's disease. Brain, 126, 713-723.

Aron, A.R., Poldrack, R.A. \& Wise, S.P. (2009) Cognition: basal ganglia role. In Squire, L.R. (Ed.), Encyclopedia of Neuroscience. Academic Press, Oxford, pp. 1069-1077.

Botvinick, M., Nystrom, L.E., Fissel, K., Carter, C.S. \& Cohen, D. (1999) Conflict monitoring versus selection-for-action in anterior cingulate cortex. Nature, 402, 179-181.

Collette, F., Hogge, M., Salmon, E. \& Van der Linden, M. (2006) Exploration of the neural substrates of executive functioning by functional neuroimaging. Neuroscience, 139, 209-221.

Coulthard, E.J., Nachev, P. \& Husain, M. (2008a) Control over conflict during movement preparation: role of posterior parietal cortex. Neuron, 58, 144-157.

Coulthard, E.J., Rudd, A. \& Husain, M. (2008b) Motor neglect associated with loss of action inhibition. J. Neurol. Neurosurg. Psychiatry, 79, 1401-1404.

Dehaene, S., Naccache, L., Le Clec'H, G., Koechlin, E., Mueller, M., DehaeneLambertz, G., van de Moortele, P.F. \& Le Bihan, D. (1998) Imaging unconscious semantic priming. Nature, 395, 597-600.

Dehaene, S., Naccache, L., Cohen, L., Bihan, D.L., Mangin, J.F., Poline, J.B. \& Rivière, D. (2001) Cerebral mechanisms of word masking and unconscious repetition priming. Nat. Neurosci., 4, 752-758.

Dehaene, S., Artiges, E., Naccache, L., Martelli, C., Viard, A., Schurhoff, F., Recasens, C., Paillère-Martinot, M.-L., Leboyer, M. \& Martinot, J. (2003) Conscious and subliminal conflicts in normal subjects and patients with schizophrenia: the role of the anterior cingulate. Proc. Natl Acad. Sci. USA, 100, 13722-13727.

D'Ostilio, K. \& Garraux, G. (2011) Automatic stimulus-induced medial premotor cortex activation without perception or action. PLOS ONE, 6, e16613.

Eimer, M. \& Schlaghecken, F. (1998) Effects of masked stimuli on motor activation: behavioral and electrophysiological evidence. J. Exp. Psychol. Hum. Percept. Perform., 24, 1737-1747.

Eriksen, B.A. \& Eriksen, C.W. (1974) Effects of noise letters upon the identification of a target letter in a nonsearch task. Percept. Psychophys., 16, 143-149.

Gajewski, P.D., Stoerig, P. \& Falkenstein, M. (2008) ERP - correlates of response selection in a response conflict paradigm. Brain Res., 1189, 127-134.

Gehring, W.J., Goss, B., Coles, M.G.H., Meyer, D.E. \& Donchin, E.A. (1993) A neural system for error-detection and compensation. Psychol. Sci., 4, 385390.

Grafton, S.T. \& Hamilton, C. (2007) Evidence for a distributed hierarchy of action representation in the brain. Hum. Mov. Sci., 26, 590-616.

Hamilton, A.F. \& Grafton, S.T. (2009) Repetition suppression for performed hand gestures revealed by fMRI. Hum. Brain Mapp., 30, 2898-2906.

Hazeltine, E., Poldrack, R. \& Gabrielli, J.D.E. (2000) Neural activation during response competition. J. Cogn. Neurosci., 12, 118-129.

Henson, R.N. \& Rugg, M.D. (2003) Neural response suppression, haemodynamic repetition effects, and behavioural priming. Neuropsychologia, 41, 263-270.

Horner, A.J. \& Henson, R.N. (2008) Priming, response learning and repetition suppression. Neuropsychologia, 46, 1979-1991.
Hughes, G., Velmans, M. \& De Fockert, J. (2009) Unconscious priming of a no-go response. Psychophysiology, 46, 1258-1269.

Klopp, B., Mattler, U., Goertz, R. \& Rist, F. (1996) N2, P3 and the lateralized readiness potential in a nogo task involving selective response priming. Electroencephalogr. Clin. Neurophysiol., 99, 19-27.

Kouider, S., Dehaene, S., Jobert, A. \& Le Bihan, D. (2007) Cerebral bases of subliminal and supraliminal priming during reading. Cereb. Cortex, 17, 2019-2029.

Kouider, S., de Gardelle, V., Dehaene, S., Dupoux, E. \& Pallier, C. (2010) Cerebral bases of subliminal speech priming. Neuroimage, 49, 922-929.

Lau, H.C. \& Passingham, R.E. (2007) Unconscious activation of the cognitive control system in the human prefrontal cortex. J. Neurosci., 27, $5805-5811$.

Lau, H.C., Rogers, R.D. \& Passingham, R.E. (2006) Dissociating response selection and conflict in the medial frontal surface. Neuroimage, 29, 446451.

MacDonald, A.W., Cohen, J.D., Stenger, V.A. \& Carter, C.S. (2000) Dissociating the role of the dorsolateral prefrontal and anterior cingulate cortex in cognitive control. Science, 288, 1835-1838.

Nee, D.E., Wager, T.D. \& Jonides, J. (2007) Interference resolution: insights from a meta-analysis of neuroimaging tasks. Cogn. Affect. Behav. Neurosci., 7, 1-17.

Neumann, O. (1990) Direct parameter specification and the concept of perception. Psychol. Res., 52, 207-215.

Neumann, O. \& Klotz, W. (1994) Motor response to nonreportable, masked stimuli: where is the limit of direct parameter specification? In Moscovitch, M. \& Umiltá, C. (Eds), Attention \& Performance XV: Conscious and Unconscious Information Processing. MIT Press, Cambridge, MA, pp. 123150

Norman, D.A. \& Shallice, T. (1986) Attention to action: willed and automatic control of behavior. In Davidson, R.J., Schwartz, G.E. \& Shapiro, D. (Eds) Consciousness and Self Regulation: Advances in Research and Theory. Plenum Press, New York, NY, pp. 1-18.

Pavone, E.F., Marzi, A. \& Girelli, M. (2009) Does subliminal visual perception have an error-monitoring system? Eur. J. Neurosci., 30, 1424-1431.

Penny, W.D., Holmes, A.P. \& Friston, K.J. (2003) Random effects analysis. In Frackowiak, R.S.J., Friston, K.J., Frith, C., Dolan, R., Friston, J., Price, C.J., Zeki, S., Ashburner, J. \& Penny, W.D. (Eds), Human Brain Function, 2nd Edn. Academic Press, San Diego, CA, pp. 849-850.

Playford, E.D., Jenkins, I.H., Passingham, R.E., Nutt, J., Frackowiak, R.S.J. \& Brooks, D.J. (1992) Impaired mesial frontal and putamen activation in Parkinson's disease: a positron emission tomography study. Ann. Neurol., 32, 151-161

Praamstra, P. \& Seiss, E. (2005) The neurophysiology of response competition: motor cortex activation and inhibition following subliminal response priming. J. Cogn. Neurosci., 17, 483-493.

Praamstra, P., Plat, E.M., Meyer, A.S. \& Horstink, M. (1999) Motor cortex activation in Parkinson's disease: dissociation of electrocortical and peripheral measures of response generation. Mov. Disord., 14, 790-799.

Salimpoor, V.N., Chang, C. \& Menon, V. (2010) Neural basis of repetition priming during mathematical cognition: repetition suppression or enhancement? J. Cogn. Neurosci., 22, 790-805.

Schlaghecken, F. \& Eimer, M. (2002) Motor activation with and without inhibition: evidence for a threshold mechanism in motor control. Percept. Psychophys., 64, 148-162

Schlaghecken, F. \& Maylor, E.A. (2005) Motor control in old age: evidence of impaired low-level inhibition. J. Gerontol. B Psychol. Sci. Soc. Sci., 60, 158 161

Schlaghecken, F. \& Sisman, R. (2006) Low-level motor inhibition in children: evidence from the negative compatibility effet. Adv. Cogn. Psychol., 2, 7-19.

Seiss, E. \& Praamstra, P. (2004) The basal ganglia and inhibitory mechanisms in response selection: evidence from subliminal priming of motor responses in Parkinson's disease. Brain, 127, 330-339.

Seiss, E. \& Praamstra, P. (2006) Time-course of masked response priming and inhibition in Parkinson's disease. Neuropsychologia, 44, 869-875.

Sohrabi, A. \& West, R.L. (2009) Positive and negative congruency effects in masked priming: a neuro-computational model based on representation, attention, and conflict. Brain Res., 1289, 124-132.

Sumner, P., Nachev, P., Morris, P., Jackson, S.R., Kennard, C. \& Husain, M. (2007) Human medial frontal cortex mediates unconscious inhibition of voluntary action. Neuron, 54, 697-711.

Taylor, S.F., Stern, E.R. \& Gehring, W.J. (2007) Neural systems for error monitoring: recent findings and theoretical perspectives. Neuroscientist, 13, $160-172$. 
340 K. D’Ostilio and G. Garraux

Ursu, S., Clark, K.A., Aizenstein, H.J., Stenger, V.A. \& Carter, C.S. (2008) Conflict-related activity in the caudal anterior cingulate cortex in the absence of awareness. Biol. Psychol., 80, 279-286.

Van Gaal, S., Ridderinkhof, K.R., Fahrenfort, J.J., Scholte, H.S. \& Lamme, V.A. (2008) Frontal cortex mediates unconsciously triggered inhibitory control. J. Neurosci., 28, 8053-8062.

Van Gaal, S., Ridderinkhof, K.R., Scholte, H.S. \& Lamme, V.A. (2010) Unconscious activation of the prefrontal no-go network. J. Neurosci., 30, 4143-4150.

Van Veen, V. \& Carter, C.S. (2002) The timing of action monitoring processes in anterior cingulate cortex. J. Cogn. Neurosci., 14, 593-602.
Wager, T.D., Sylvester, C.Y.C., Lacey, S.C., Nee, D.E., Franklin, M. \& Jonides, J. (2005) Common and unique components of response inhibition revealed by fMRI. Neuroimage, 27, 323-340.

Weiskrantz, L., Warrington, E.K., Sanders, M.D. \& Marshall, J. (1974) Visual capacity in the hemianopic field following a restricted occipital ablation. Brain, 97, 709-728.

Wenke, D., Fleming, S.M. \& Haggard, P. (2010) Subliminal priming of actions influences sense of control over effects of action. Cognition, 115, 26-38.

Wylie, S.A., Stout, J.C. \& Bashore, T.R. (2005) Activation of conflicting responses in Parkinson's disease: evidence for degrading and facilitating effects on response time. Neuropsychologia, 43, 1033-1043. 